\title{
Optical Heterodyne Analog Radio-over-Fiber Link for Millimeter-Wave Wireless Systems
}

\author{
Amol Delmade, Member, IEEE, Colm Browning, Member, IEEE, Theo Verolet, Julien Poette, \\ Arman Farhang, Member, IEEE, Hamza Hallak Elwan, R David Koilpillai, Member, IEEE, \\ Guy Aubin, Senior Member, IEEE, F Lelarge, Abderrahim Ramdane, Senior Member, IEEE, \\ Deepa Venkitesh, and Liam P Barry, Senior Member, IEEE
}

\begin{abstract}
Optical heterodyne analog radio-over-fiber (A-RoF) links provide an efficient solution for future millimeter wave ( $\mathrm{mm}$ wave) wireless systems. The phase noise of the photo-generated mm-wave carrier limits the performance of such links, especially, for the transmission of low subcarrier baud rate multi-carrier signals. In this work, we present three different techniques for the compensation of the laser frequency offset $(\mathrm{FO})$ and phase noise (PN) in an optical heterodyne A-RoF system. The first approach advocates the use of an analog $\mathrm{mm}$-wave receiver; the second approach uses standard digital signal processing (DSP) algorithms, while in the third approach, the use of a photonic integrated mode locked laser (MLL) with reduced DSP is advocated. The compensation of the FO and PN with these three approaches is demonstrated by successfully transmitting a 1.95 MHz subcarrier spaced orthogonal frequency division multiplexing (OFDM) signal over a $25 \mathrm{~km} 61 \mathrm{GHz}$ mm-wave optical heterodyne A-RoF link. The advantages and limitations of these approaches are discussed in detail and with regard to recent 5G recommendations, highlighting their potential for deployment in next generation wireless systems.
\end{abstract}

Index Terms - 5G, Millimeter-Wave Communications, Radioover-Fiber, Optical Heterodyning, Fronthaul

\section{INTRODUCTION}

$\mathrm{M}$ OBILE communication networks progression towards the $5^{\text {th }}$ generation $(5 \mathrm{G})$ wireless technology stems from the advent of many data hungry applications such as virtual reality (VR), live ultra-high definition (UHD) video streaming and autonomous driving. The enhanced mobile broadband services of $5 \mathrm{G}$ promises to provide data rates up to $10 \mathrm{~Gb} / \mathrm{s}$ by using the combination of techniques such as higher-order modulation formats, massive multiple-input multiple-output (MIMO) antenna technology and wide channel bandwidth (BW), in conjunction with a centralized radio access network (C-RAN) architecture [1]. C-RAN simplifies the antenna site

This work has emanated from research supported in part by a research grant 18/EPSRC/3591, 18/SIRG/5579 from Science Foundation Ireland (SFI); 13/RC/2077, 12/RC/2276 from the European Regional Development Fund; 15/US-C2C/I3132 from SFI US-Ireland Partnership Program; Irish Research Council; Ulysses Mobility programme from French Ministry of Foreign Affairs and VAJRA faculty programme of MHRD, Government of India.

(Corresponding author: Amol Delmade.E-mail: amol.delmade2@mail.dcu.ie) A. Delmade, C. Browning, and L. P. Barry are with the School of Electronic Engineering, Dublin City University, Dublin D09, Ireland

A. Farhang is with the Department of Electronic Engineering, Maynooth University, Maynooth, Ireland. architecture by moving the baseband processing unit (BBU) of a traditional base station (BS) to a centralized location, termed as centralized-BBU (C-BBU), and connecting it to the antenna site remote radio head (RRH) by an optical fiber based fronthaul link [2].

It is profoundly difficult to allocate the wider channel BWs for some $5 \mathrm{G}$ services given the spectral scarcity in the current wireless networks. This has led to the emergence of millimeterwave (mm-wave) communications - which can facilitate wider channel bandwidths by moving the carrier to the $30-300 \mathrm{GHz}$ frequency band - as a key enabling technology for $5 \mathrm{G}$ wireless systems, and beyond [3]. Simple and cost-efficient techniques, with minimal power consumption, must be employed for the generation and distribution of $\mathrm{mm}$-wave carrier. The higher losses associated with wireless propagation of $\mathrm{mm}$-wave carriers will result in the need for ultra-dense (UD) deployment of the RRHs; requiring spectrally and cost-efficient back/fronthaul links for data delivery to and from these antenna sites [3]. An efficient fronthaul network and mm-wave generation technique are two key elements for future high speed mobile communication systems.

Optical fronthaul transmission between C-BBU and RRHs is typically carried through the digitized radio-over-fiber (D-RoF) scheme used in currently deployed common public radio interface (CPRI) links; which hinders network scaling due to its poor spectral efficiency and remote hardware requirements [4]. The enhanced CPRI (e-CPRI) [5] relaxes the BW requirements over fronthaul link, to some extent, using the functional split of the radio access network; resulting in more digital functions being moved to the remote RRH antenna sites [6]. The tradeoff in the data rate requirements over the fiber link, complexity of the RRH antenna site and cost of high-speed digital functionality implementation (due to the choice of RAN split point) may result in operator and/or use case specific fronthaul networks as $5 \mathrm{G}$ evolves towards higher bandwidths.

H. H. Elwan, and J. Poette, are with the Institute of Microelectronics, Electromagnetism and Photonics, Grenoble Alpes University, France.

H. H. Elwan is also with the Orange Labs, France.

Theo Verolet, Guy Aubin, and Abderrahim Ramdane are with the Centre de Nanosciences et de Nanotechnologies, CNRS, Université Paris-Saclay, France. F Lelarge and Theo Verolet are with the III-V Lab, a joint lab of Nokia, Thales and CEA, 1, Avenue A. Fresnel, 91767 Palaiseau Cedex, France.

F Lelarge is also with the Almae Technologies, Route de Nozay, France.

R David Koilpillai and Deepa Venkitesh are with Department of Electrical Engineering, IIT Madras, India. 
The poor spectral efficiency and scalability issues of digital RoF fronthauling can be alleviated by analog radio-over-fiber (A-RoF) links [7]. An analog RoF approach retains the inherent bandwidth efficiency of the wireless signals over the fronthaul link and simplifies the RRH antenna site architecture by providing RF signals which are ready for antenna transmission directly after photo-detection and amplification. It also avoids the use of a high-speed digital-to-analog (DAC) and analog-todigital conversion (ADC) at the RRH antenna site. Various experiments [7] - [11] have demonstrated the feasibility of ARoF fronthaul supporting the transmission of multiple signal bands over the same link.

The complexity associated with the electronic generation of the $\mathrm{mm}$-wave carriers hinders its deployment in future mobile networks and hence photonic generation techniques have been proposed. Optical heterodyning, wherein two optical carriers with a spacing equal to the desired RF carrier frequency beat on a high-speed photodetector, has been studied extensively for mm-wave generation [12]-[19]. The optically compatible ARoF fronthaul network can facilitate the distribution of $\mathrm{mm}$ wave carriers to various RRH sites through the same fiber network leading the way for centralization of the resources. However, in such systems, the phase noise (PN) of the photogenerated mm-wave carriers has been shown to be a performance limiting factor [18], especially for the small baud rate/subcarrier spacing multicarrier signals as envisioned in $5 \mathrm{G}$ and beyond $5 \mathrm{G}$ wireless systems. Hence, it is necessary to either reduce the phase noise of $\mathrm{mm}$-wave carriers or compensate it in some way for the successful system implementation.

Most of the previous optical heterodyne demonstrations have used either two free running lasers [12]-[15] or optical frequency combs (OFC) [16]-[19] as optical sources for mmwave generation. In optical heterodyning systems with free running lasers, the frequency stability and linewidth of the lasers contribute to the phase noise of the photo-generated $\mathrm{mm}$ wave carriers. Techniques such as the use of dual mode lasers [15], optical injection locking [20], optical phase locked loops [21] and RF envelope detectors [14] have been employed to increase the stability and reduce the phase noise of the optical tones, but at the cost of increased optical/electrical complexity. In this paper, we present three different techniques to mitigate the effect of laser frequency fluctuations (i.e. frequency offset (FO)) and phase noise/linewidth on the system performance. The first approach advocates the use of an analog mm-wave receiver [22]; the second approach uses the standard digital signal processing (DSP) algorithms [23], while in the third approach, the use of a photonic integrated mode locked laser (MLL) with reduced DSP compensation [24] is presented.

In the following sections, we will demonstrate and discuss how these three schemes mitigate the effect of FO \& PN in an optical heterodyne A-RoF link by presenting the successful transmission of 64-QAM modulated $1.95 \mathrm{MHz}$ subcarrier spaced orthogonal frequency division multiplexing (OFDM) signals over $25 \mathrm{~km}$ standard single mode fiber (SSMF) link. Two different sets of optical sources, i.e. free running lasers and MLL OFC are used in the respective schemes. Details of the general heterodyne system implementation are discussed in Section II, while the compensation techniques and their respective results are discussed in subsections of Section III. The advantages and limitations of these techniques are discussed, with regard to the transmission of the $5 \mathrm{G}$ envisioned subcarrier spaced signals, in Section IV. Finally, Section V concludes the paper.

\section{HETERODYNE SYSTEM IMPLEMENTATION}

The schematic of the general optical heterodyne mm-wave A-RoF fronthaul link experimental setup (with representative spectra along the transmission path) is shown in Fig. 1. Two optical tones with frequency spacing $\left(\Delta \mathrm{F}_{\mathrm{c}}\right)$ close to the desired $\mathrm{mm}$-wave carrier frequency, $56 \mathrm{GHz}$ in this case, from the optical source assembly are fed to the Ch. 1 and Ch. 2 of the transmitter. As mentioned in Section I, two different optical sources i.e. either two free running tunable lasers or a passively mode lock laser based OFC followed by a filter, are used alternatively in our schemes. In the case of two free running tunable lasers (TL1 \& TL2), the output of lasers can be directly fed to channel 1 and channel 2, respectively, as shown in the inset (a) of Fig. 1, while the output of the MLL OFC needs to

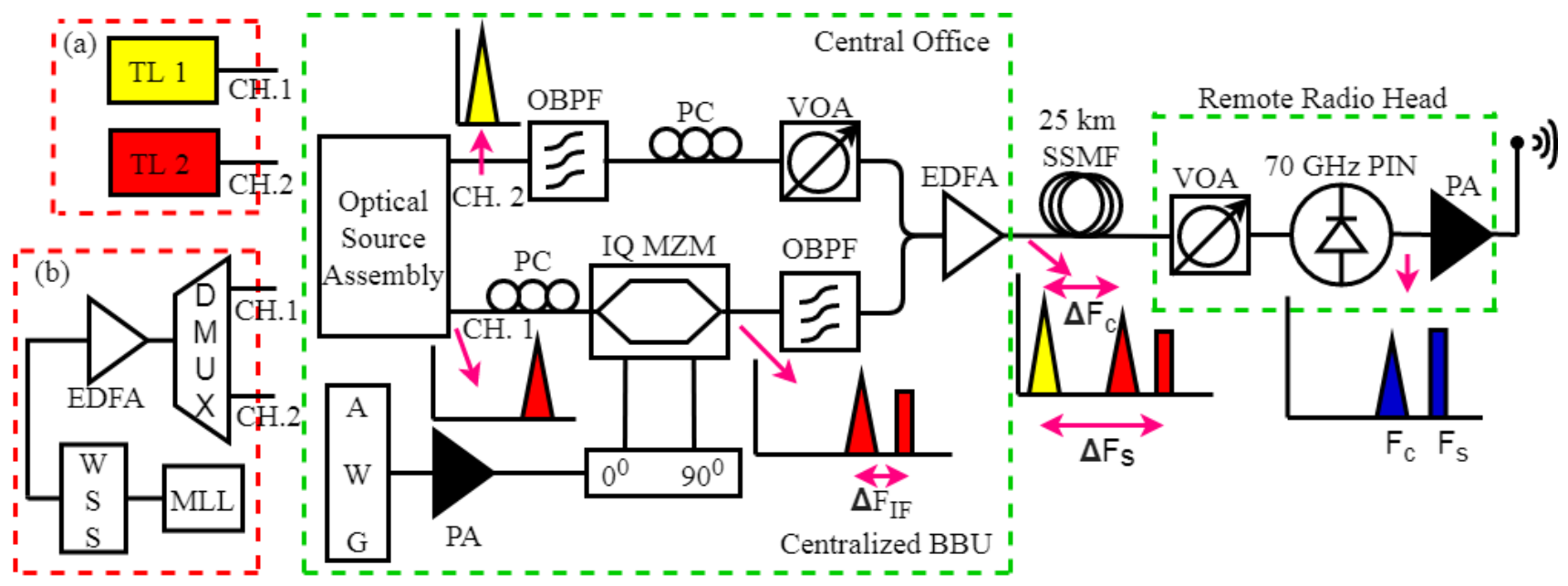

Fig. 1. Optical heterodyne mm-wave A-RoF fronthaul transmission system showing the C-BBU and RRH site architectures along with the optical source assembly insets (a) two free running lasers, (b) QD MLL OFC. 


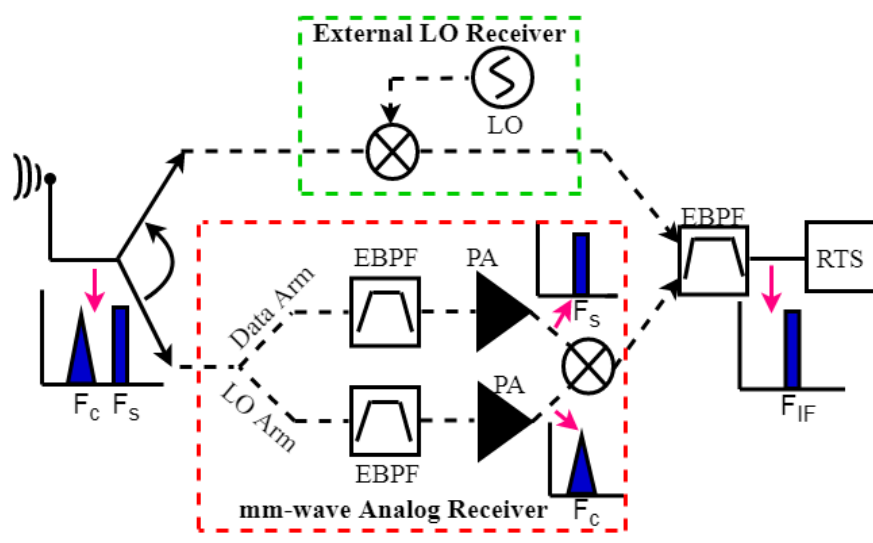

Fig. 2 Receiver architecture of the mm-wave A-RoF system showing two different sets of frequency down-conversion mechanism in (a) red box analog mm-wave stage and (b) green box - external standalone LO stage.

be filtered before feeding it to the two respective input channels. A wavelength selective switch (WSS) is used to select two optical tones from the output of the MLL OFC and is followed by an erbium doped fiber amplifier (EDFA) to boost the power of these carriers before splitting the optical path to two channels as shown in the Inset (b) of Fig. 1. More details of the tunable lasers and MLL are discussed in respective sections.

An optical single sideband (OSSB) modulated OFDM signal (red signal in Fig. 1) is generated using an I/Q Mach-Zehnder Modulator and electrical $90^{\circ}$ hybrid coupler in Ch. 1. The OFDM signal was generated in the electrical domain, at an intermediate frequency (IF) of $5 \mathrm{GHz}\left(\mathrm{F}_{\mathrm{IF}}\right)$, using an arbitrary waveform generator (AWG). The OFDM signal consists of 64QAM modulated subcarriers at a spacing of $1.95 \mathrm{MHz}$. The OSSB modulated carrier was combined with the un-modulated carrier from Ch. 2 (yellow carrier in Fig. 1) and transmitted through $25 \mathrm{~km}$ of SSMF after amplification by an EDFA. A variable optical attenuator (VOA) was used in Ch. 2 to equalize the optical power between the two optical paths due to different losses. Optical band pass filters (OBPF) are used in both paths to remove the unwanted signal or carrier components.

Two optical tones, separated by $56 \mathrm{GHz}$, and a data signal which is modulated $5 \mathrm{GHz}$ away from one of the carrier tones, are transmitted together through the fiber to the RRH site transmit antenna. The photo-mixing of these three optical frequency components, on a $70 \mathrm{GHz}$ PIN PD, generates the electronic copies of the mm-wave OFDM data signal at $\mathrm{Fs}_{\mathrm{s}}=61$ $\mathrm{GHz}$ (from un-modulated carrier $\times$ OSSB data sideband mixing) along with a mm-wave carrier at $\mathrm{F}_{\mathrm{C}}=56 \mathrm{GHz}$ (from carrier $\times$ carrier mixing). The strength of the generated mmwave carrier and data signal will depend on the powers of both modulated and un-modulated optical carriers as well as OSSB modulated data signal. If the modulated carrier (red carrier in Fig. 1) remains un-suppressed after OSSB modulation, then there will be a relatively strong beating term between this carrier and un-modulated carrier (yellow) - producing a relatively high powered mm-wave carrier at $\mathrm{Fc}$ at the $\mathrm{PD}$ output. A VOA at the RRH site is used to control the total power incident on the high-speed PD.

The photo-generated $61 \mathrm{GHz}$ OFDM signal can be directly transmitted to the wireless user using mm-wave antenna elements, after amplification, as shown in RRH box of Fig. 1. It is worth mentioning that wireless transmission of mm-wave signal was not carried in this experiment. The output of PD was directly connected to the input of frequency down-conversion stage of receiver presented in Fig. 2.

The three techniques presented in this paper use two different approaches for remote frequency down-conversion of the 61 $\mathrm{GHz}$ mm-wave OFDM signal to IF, as shown in the two arms of the receiver architecture of Fig. 2. The analog mm-wave receiver approach (red box in Fig. 2) uses the photo-generated RF carrier itself for frequency down-conversion of the signal, requiring the transmission of both mm-wave signal and carrier over the wireless channel. The second receiver scheme (green box in Fig. 2) employs an external local oscillator (LO) and mixer for mm-wave signal frequency down-conversion to IF. The minimum power level, of the IF signal, on the receiver side was limited to $-28 \mathrm{dBm}$ due to the significant effect of quantization noise introduced by the RTS below this power. More details of this receiver structure are discussed in the respective receiver sections. A real time oscilloscope (RTS) was used to capture the down converted IF data. Offline processing is applied on the captured signal, using Matlab, to evaluate the bit error rate (BER) and error vector magnitude (EVM) performance.

This system architecture is designed to replicate the future mobile fronthaul link wherein analog RoF signals are transmitted from a central office $(\mathrm{CO})$ or C-BBU through 25 $\mathrm{km}$ of SSMF to the RRH site, where the frequency upconversion of the data to mm-wave band is performed by optical heterodyning of relevant optical data signal and carrier.

\section{FREQUENCY OFFSET AND PHASE NOISE COMPENSATION TECHNIQUES}

\section{A. MM-WAVE ANALOG RECEIVER}

\section{1) $F O \& P N$ Cancellation}

The photo-mixing, at the RRH site, generates the electronic copies of mm-wave OFDM data signal at $\mathrm{Fs}=61 \mathrm{GHz}$ and a mm-wave carrier at $\mathrm{FC}_{\mathrm{C}}=56 \mathrm{GHz}$. The frequency drift of the free running lasers (TL1 \& TL2) will result in the equal amount of frequency shift (say $\delta \mathrm{F}$ ) in both $\mathrm{F}_{\mathrm{C}}$ (will become $\mathrm{F}_{\mathrm{C}} \pm \delta \mathrm{F}$ ) and Fs (will become Fs $\pm \delta F$ ), after the photo-detection. The frequency shift, $\delta \mathrm{F}$, changes over time, but the difference

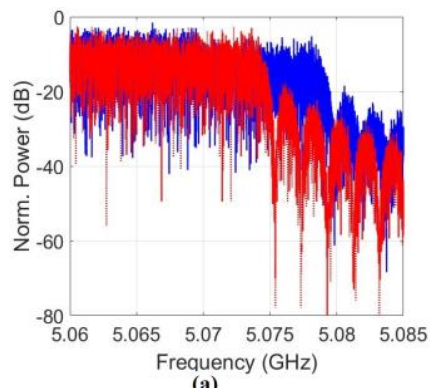

(a)

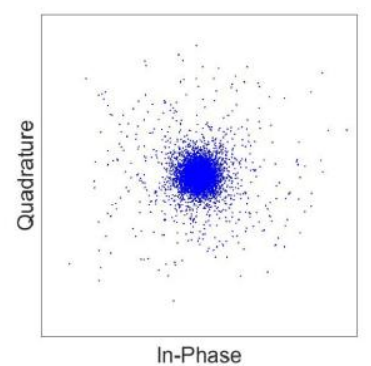

(b)
Fig. 3(a) A portion of the transmitted (red) and received (blue) IF signal spectra showing non-overlap due to FO and (b) Constellation of the demodulated signal in an optical heterodyne A-RoF link with free running lasers source and fixed frequency external LO down-conversion receiver. 
between $F_{s}$ and $F_{C}$ will remain equal to $F_{I F}$. The frequency down-conversion of such a mm-wave OFDM data signal, centered at $\mathrm{Fs} \pm \delta \mathrm{F}$, using a fixed frequency external LO (as shown in the green box portion of receiver in Fig. 2) will result in the frequency shifted IF signal, with a spectrum as presented in Fig. 3(a). Demodulation of this frequency shifted IF signal, after capturing and processing using Matlab, will result in the distorted constellation, due to FO and PN, as shown in Fig. 3(b). Hence, the external LO used for down conversion of the mmwave OFDM data signal to $5 \mathrm{GHz}$ IF, needs to replicate the laser frequency drift over time in order to recover the data.

Regardless of the frequency drift of free running tunable lasers, the difference between $\mathrm{FC}_{\mathrm{C}} \pm \delta \mathrm{F}$ and $\mathrm{Fs} \pm \delta \mathrm{F}$ terms will always be equal to $5 \mathrm{GHz}$, which is the original IF. Hence, the mixing of these two photo-generated mm-wave components can lead to the frequency down-conversion of the mm-wave data signal $(\mathrm{Fs} \pm \delta \mathrm{F})$ to original $5 \mathrm{GHz}$ intermediate frequency; eventually making the system agnostic to the laser frequency drift. Achieving robustness to FO in this way negates the requirement for frequency correlation, or locking, between the laser sources - significantly reducing transmitter complexity. Also, this signifies that the generated $\mathrm{mm}$-wave carrier at $\mathrm{FC}_{\mathrm{C}} \pm$ $\delta \mathrm{F}$ can be used as an external LO for the frequency downconversion of the mm-wave data signal; achieving an external 'LO-free' frequency down-conversion. Eventually, this will necessitate the transmission of both $\mathrm{mm}$-wave signal and carrier over the wireless channel. This concept has led to the design of our mm-wave analog receiver, which is shown in the red box of Fig. 2.

The signal captured by the antenna is split into two paths, the LO arm and data arms, to separate the mm-wave carrier and $\mathrm{mm}$-wave data signal, respectively, by using electrical band pass filters (EBPF). The center frequencies and $3 \mathrm{~dB}$ bandwidths of both EBPF: $f=56 \mathrm{GHz}, B W=500 \mathrm{MHz}(\mathrm{LO}$ arm) and $f=$ $62 \mathrm{GHz}, B W=6 \mathrm{GHz}$ (RF data arm), are chosen considering the IF carrier and frequency drifts of the commercially available tunable lasers. The filtered signals are mixed together using a $\mathrm{mm}$-wave mixer; resulting in a data signal output at IF of 5 $\mathrm{GHz}$. This IF signal is captured using an RTS, and processed in Matlab, as shown in the receiver architecture of Fig. 2.

It is worth mentioning that the photo-mixing generated $\mathrm{mm}$ wave carrier will have the same phase noise as that of the $\mathrm{mm}$ wave data signal. Mixing of these phase noise correlated mmwave frequency components, will result in the cancellation of the RF phase noise, of the IF signal, upon frequency down-

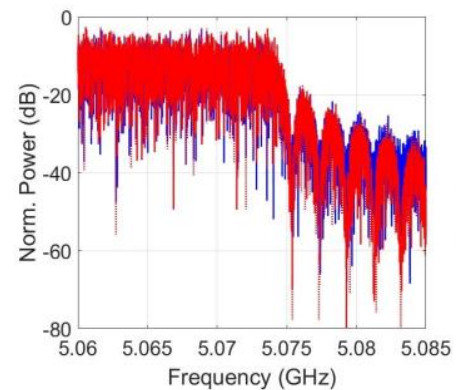

(a)

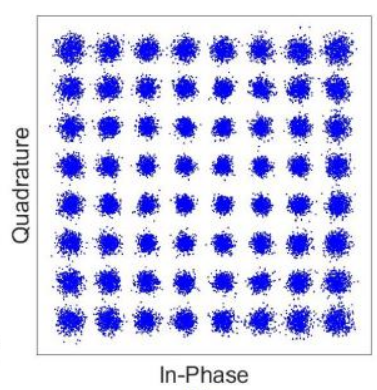

(b)
Fig. 4(a) A portion of the transmitted (red) and received (blue) IF signal spectra showing exact overlap and (b) Constellation of the demodulated signal in an optical heterodyne A-RoF link with free running lasers source and standalone analog $\mathrm{mm}$-wave receiver. conversion. Achieving phase noise cancellation, in this manner, effectively removes the requirement for phase correlated or ultra-low linewidth laser sources which have been shown to be a necessity for heterodyne A-RoF applications [18]. The presented architecture permits a significant reduction in the complexity of the C-BBU transmitter and RRH antenna site designs. It should be noted that these advantages come at the cost of a requirement for the beat term to be transmitted over wireless channel (after the PD) in tandem with the data signal.

The previous system demonstrations [25], [26] use a similar electronic phase noise cancellation mechanism, but the requirement of a correlated dual-mode optical source, for the de-multiplexing and cancellation operation, increases the receiver unit's optical complexity. A similar concept is demonstrated in [27] albeit with the use of square law nonlinear device which potentially puts additional constraints on input (received) power.

\section{2) Key Results}

Initially, the mm-wave A-RoF heterodyne system was tested with two hybrid InP-TriPleX photonic integrated tunable lasers [28] at the transmitter and a standalone $56 \mathrm{GHz}$ external $\mathrm{LO}$ at the receiver. The lasers exhibit excellent linewidth of $\sim 50 \mathrm{kHz}$ and side mode suppression ratio (SMSR) of $<50 \mathrm{~dB}$. An universally filtered OFDM signal consisting of 76 subcarriers with 64-QAM data symbols at a spacing of $1.95 \mathrm{MHz}$ was used. It resulted in a signal BW of $148 \mathrm{MHz}$ and raw data rate of 0.89 $\mathrm{Gb} / \mathrm{s}$. Fig. 3(a) shows the frequency offset between the nonoverlapping spectra of the transmitted (red) and received (blue) IF signal. A maximum carrier frequency offset/spectrum nonoverlap, due to the relative frequency drift of the TLs, of $\sim 10$ $\mathrm{MHz}$ was observed over several captures. As mentioned earlier, the captured OFDM signal could not be demodulated successfully due to the combined effects of FO and PN, resulting in the received noisy constellation presented in Fig. 3(b).

The standalone external $\mathrm{LO}$ receiver was replaced by the $\mathrm{mm}$ wave analog receiver in order to test its capabilities for FO \& PN compensation. Fig. 4(a) shows the spectra of transmitted (red) and received (blue) IF signal in this case. A perfect overlap between a portion of the transmitted and received IF signal electrical spectra indicates that the system is agnostic to the frequency fluctuations between the two TLs. Optical heterodyning, even with two low-linewidth $(50 \mathrm{kHz}) \mathrm{TLs}$, produces a mm-wave data signal with insufficient purity in order to support 64-QAM subcarrier modulation at 1.95 Mbaud rate [8]. However, by utilizing the proposed receiver architecture, mm-wave phase noise cancellation (PNC) is enabled. The constellation of the received signal, shown in Fig. 4(b), indicates the compensation of the signal phase noise and results in the successful demodulation of the OFDM signal. In this case the received EVM was $5.8 \%$ with BER measured to be $7.4 \times 10^{-5}$.

These experimental results highlight the proposed system's robustness to both phase noise and frequency offset, while facilitating a reduction in optical complexity of the A-RoF heterodyne system - arising from the use of unlocked and uncorrelated free-running lasers. The implementation of the analog receiver clearly adds complexity at the mm-wave receiver side, and it must be mentioned that current mobile 
communication systems do not have a provision for the transmission of a mm-wave carrier over the air. However, as deployed networks evolve to exploit the mm-wave technologies, the costs associated with the remote carrier generation at the receiver side makes the concept of a centralized carrier distribution attractive. Such a scheme can be effectively implemented through the use of the $\mathrm{mm}$-wave analog receiver. The compatibility of this approach for the beamforming need to be studied for achieving high data rate transmission.

\section{B. DSP RECEIVER}

In the DSP receiver [23] approach, the standard 'Schmidl and Cox' (S\&C) [29] and decision directed least mean square (DDLMS) algorithms are used for FO \& PN compensation, respectively. The implementation of this DSP receiver will not require any additional processing as these algorithms have been traditionally implemented in the wireless systems for RF FO \& PN compensation at the mobile receivers. Robustness to timing and frequency offsets through digital means is a well-explored topic for high baud rate optically coherent OFDM systems [30], [31], which are inherently more tolerant to frequency and phase offsets compared to A-RoF systems.

In the absence of a feedback mechanism to track the laser frequency fluctuations (or inability to transmit the photomixing generated carrier as presented earlier) a fixed frequency LO along with FO \& PN compensation techniques based on digital signal processing at the receiver side, can lead to a simple and cost efficient mm-wave A-RoF system design. In this scenario, the electronic complexity of the analog mm-wave receiver demonstrated in the previous section is transferred to the digital domain. The fronthaul link and transmitter architecture of this scheme remains the same as shown in Fig. 1 , with the exception that the photo-mixing generated $\mathrm{mm}$-wave carrier is suppressed; while the receiver uses an external mm-

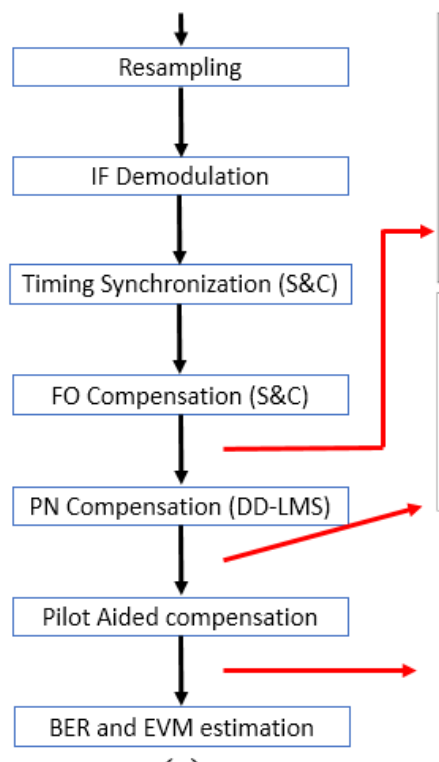

(a)

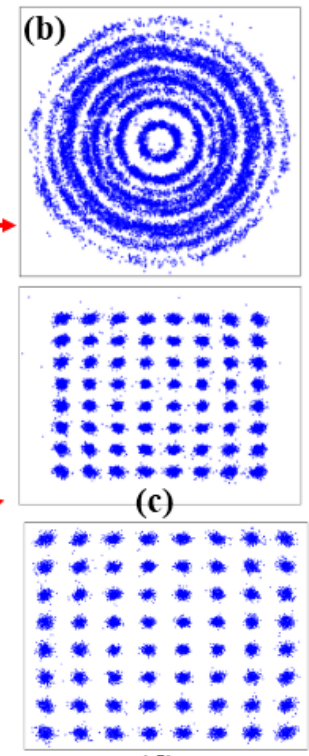

(d)
Fig. 5(a) The flow chart of offline DSP operations for demodulation of the signal; Constellation of the received signal after (b) FO compensation only (c) FO \& PN compensation and (d) all the DSP processing. wave LO for remote frequency down-conversion, as shown in the portion of the receiver structure outlined in green in Fig. 2.

In this case, two low noise fiber lasers, each with a linewidth of $\sim 1 \mathrm{kHz}$, were used at the transmitter. A $195 \mathrm{MHz}$ bandwidth OFDM signal carrying a raw data rate of $1.12 \mathrm{~Gb} / \mathrm{s}$ was modulated. The signal consists of 100 subcarriers, each with 64QAM data symbols, at a spacing of $1.95 \mathrm{MHz}$.

\section{1) $F O \& P N$ compensation}

In the DSP receiver architecture scheme, the received mmwave data signal at the user unit side is down converted, to an intermediate frequency band, by mixing with a free running external LO. The RTS captures this signal and resamples it before processing for BER and EVM performance analysis. The flow chart of the steps involved in our offline processing is shown in the Fig. 5(a). The spectrum of the resampled received IF signal resembles the one shown in Fig. 3(a), as relative frequency drift between the lasers prevents OFDM demodulation. This OFDM signal, centered at $5 \mathrm{GHz} \pm \delta \mathrm{F}$, is then frequency down converted to the baseband by mixing it with a $5 \mathrm{GHz}$ IF carrier in Matlab. S\&C and DD-LMS algorithms were then implemented digitally to help overcome FO \& PN, respectively. Aside from the phase noise, resulting from the linewidths of the transmitter lasers, the system will also produce random relative phase variations between the external $\mathrm{LO}$ and the received photo-generated $\mathrm{mm}$-wave signal. This additional $\mathrm{PN}$ also needs to be compensated for the reliable detection of the transmitted data.

Timing synchronization and FO estimation in the frequency down converted baseband signal is achieved by searching for a OFDM symbol with two identical halves. This special preamble, as defined in S\&C algorithm, is transmitted at the start of the OFDM data from transmitter. The integer (subcarrier index) FO is detected by cross correlation of the transmitted and received preamble, instead of using a second preamble as described in $\mathrm{S} \& \mathrm{C}$ algorithm. The detected data is counter rotated in the frequency domain by a number of samples equal to the integer FO to correct for the FO. The same preamble is used for channel estimation. The fractional FO, which is less than the OFDM subcarrier spacing, is manifested as phase rotation in the constellation.

Fig. 5(b) shows the constellation of the OFDM demodulated signal immediately after the FO compensation. This phase rotated constellation indicates the presence of phase noise in the signal, which needs to be corrected for successful system implementation. A DD-LMS approach was implemented in order to track phase rotation with respect to the ideal constellation diagram. Fig. 5(c) shows the constellation of the OFDM demodulated signal after FO and DD-LMS PN compensation. The phase ambiguity which results from such blind equalization was compensated for by the inclusion of several pilot subcarriers which were used to estimate phase shifts across the occupied OFDM spectrum. Fig. 5(d) shows the constellation of the OFDM demodulated signal after employing all necessary digital post-processing operations.

\section{2) Key Results}

The constellation results shown in Fig. 5 clearly indicate the capabilities of the DSP receiver to compensate the FO and PN completely. For a received optical power of $-2 \mathrm{dBm}$, an EVM 
of $4.76 \%\left(\mathrm{BER}=3.13 \times 10^{-5}\right)$ is achieved and performance is within the forward error correction (FEC) limit for the received power as low as $-8 \mathrm{dBm}$. Our recent work, presented in [32], shows that there is no penalty due to $25 \mathrm{~km}$ of fiber transmission over back-to-back transmission. Received optical power variations from $-2 \mathrm{dBm}$ to $-10 \mathrm{dBm}$ result in EVM variation from $4.76 \%$ to $\sim 12 \%$ [32]. The excellent performance obtained at these lower power levels is attributed to the good quality of fiber lasers, with SMSR $>50 \mathrm{~dB}$, used for this experiment.

Also, in the recent work [23] with this DSP receiver we demonstrated the successful transmission of a $61 \mathrm{GHz}$ OFDM signals with subcarrier baud rates as low as $125 \mathrm{kHz}$ - which is envisioned in the latest $5 \mathrm{G}$ new radio specifications. The frequency offsets of up to $140 \mathrm{MHz}$ are compensated by using this digital processing without any significant performance degradation [23] - indicating the suitability of this approach for future hybrid optical/mm-wave multicarrier transmission systems. The limitation of time varying frequency shift of the $\mathrm{mm}$-wave signal can be eliminated by using commercially available lasers, whose frequency drift is limited within the guard bands of the wireless channels. Hence, the utilization of established DSP with 'off-the-shelf' commercial lasers paves the way for the deployment of a low baud rate multicarrier signals carrying optical heterodyne mm-wave A-RoF system.

\section{OFC \& Reduced DSP Receiver}

The frequency offset, in the optical heterodyne system, resulting from the frequency fluctuations of the free running lasers can be eliminated by replacing the sources with an optical frequency comb. The use of OFCs, for mm-wave signal generation, gives precise control over the center frequency of the mm-wave signal to be transmitted from the antenna unit. Previous work on OFC based optical heterodyning analog RoF [18] has shown how the coherence length of correlated optical tones requires strict path matching between these carriers throughout the system. Effective path length difference and fiber dispersion in the system results in decorrelation at the receiver - producing mm-wave carriers with relatively high levels of phase noise. This essentially necessitates the use of a low linewidth $(<$ few MHz) and highly correlated OFC source for the flexible implementation of the optical heterodyne $\mathrm{mm}$ wave A-RoF system with relaxed path matching constraints.

Our previous work in [18], successfully demonstrated such a system with a gain switched laser (GSL) OFC for FO compensation and analog $\mathrm{mm}$-wave receiver, described in section III.A, for PN compensation. The use of high-power RF source for gain switching along with the requirement of a low linewidth laser for external injection to the GSL (to reduce phase noise on the comb lines) [33], increases the complexity of the optical heterodyning system. In this present work, an RF synthesizer free low linewidth photonic integrated quantum dash passively mode locked laser (QD-P-MLL) OFC is used to meet the necessary laser specifications and cost-effectiveness for wide deployment. To relax the constraints on the path matching and correlation of OFC tones, our approach uses digital PN compensation algorithm at the receiver along with low linewidth MLL as optical source. Previous work with a MLL [34] has demonstrated the transmission of high baud rate single carrier signals, for which the impact of phase noise is

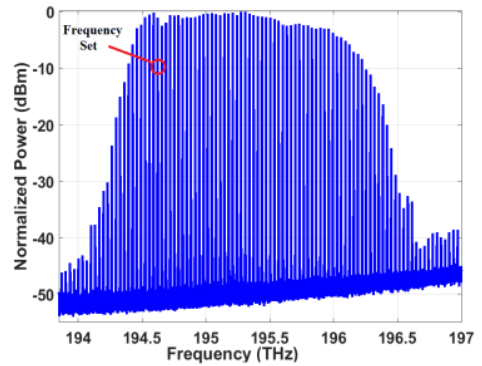

(a)

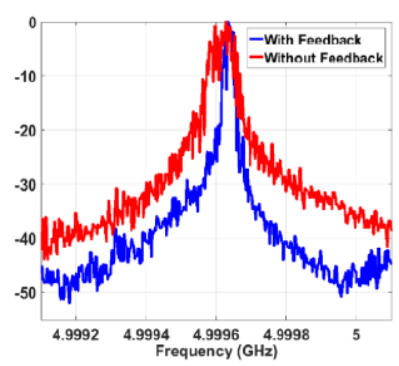

(b)
Fig. 6 (a) The optical spectrum at the output of passively mode locked QD laser with red circles showing different sets of operating frequencies used for the heterodyning and (b) the RF spectrum of the frequency down converted mm-wave carrier, in the system, for MLL with and without feedback case.

minimal. Here, we have demonstrated the transmission of $5 \mathrm{G}$ envisioned small subcarrier spacing multi-carrier signals.

\section{1) QD-P-MLL Source \& System Description}

An integrated single section Fabry-Pérot cavity mode lock laser with a repetition rate of $32.5 \mathrm{GHz}$ was used for this work. The gain medium of MLL was made with a few layers of InAs quantum dash, while cleaved facets were used for the mirrors. The complete description of the device can be found in [35], [36]. This OFC source fiber couples the output power of $\sim 9 \mathrm{dBm}$ and exhibits a $3 \mathrm{~dB}$ envelope bandwidth of $11.8 \mathrm{~nm}$ (spectrum shown in Fig. 6(a)) at $300 \mathrm{~mA}$ bias current. The optical linewidth of the comb tones was around $6 \mathrm{MHz}$ and the $\mathrm{RF}$ beat signal of all comb tones had a linewidth of $\sim 10 \mathrm{~s} \mathrm{kHz}$. An optical external free space cavity, consisting of a collimating lens, a free space attenuator and a mirror, is placed backwards the MLL chip to reduce the phase noise of both the optical lines and RF beat signal [37]. It introduces an optical feedback whose strength and phase are changed by tuning the position of mirror, which results in linewidth change. Under coherent feedback both the optical linewidths and RF linewidth can be reduced by a factor of 10 [38].

The output of this MLL OFC was fed to a WSS which filtered two optical tones spaced close to the required mm-wave frequency, in this case $65 \mathrm{GHz}$ (twice the FSR of the OFC), as shown in the inset (b) of Fig. 1. Two optical tones, at 194.556 and $194.621 \mathrm{THz}$, were selected for heterodyning, and are marked by the red circles on the MLL output spectrum shown in Fig. 6(a). The output of the WSS is amplified and sent to the two channels of the heterodyning architecture as shown in the inset (b) of Fig. 1. Most of the remaining details of the experimental setup are similar to that discussed in section II. With regard to the generation of the OSSB signal, the biasing conditions of the IQ MZM were changed in order to generate the lower OFDM sideband at $5 \mathrm{GHz}$ relative to the optical carrier. Given the selected tone spacing of $65 \mathrm{GHz}$, this operation allowed OFDM signal generation at $60 \mathrm{GHz}$ through the heterodyne process. In this scheme also, a $195 \mathrm{MHz}$ bandwidth OFDM signal, with a subcarrier baud rate of 1.95 Mbaud, carrying a raw data rate of $1.12 \mathrm{~Gb} / \mathrm{s}$ was transmitted over the link. The receiver architecture, for this scheme, is similar to the previous scheme presented in section III.B, but with reduced DSP for PN compensation alone. It consists of an external standalone LO for frequency down-conversion to IF and DSP algorithms for PN compensation. 


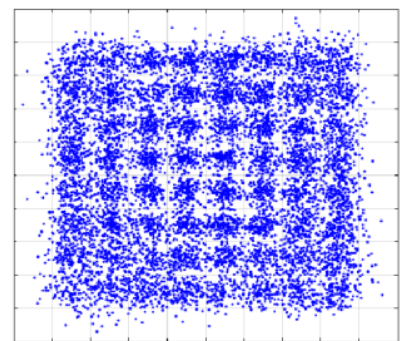

(a)

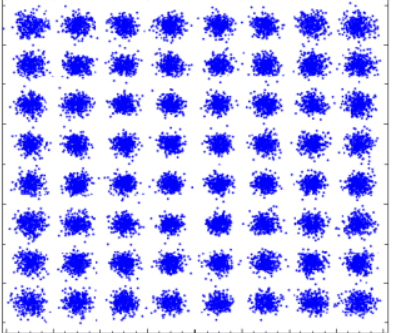

(b)
Fig. 7 Constellation of the demodulated signal in a mm-wave A-RoF system employing a QD-P-MLL OFC (a) without and (b) with a free space optical feedback.

\section{2) Results}

Initially, the optical heterodyne system was tested when there was no feedback applied to the MLL OFC. The constellation of the received demodulated signal is shown in Fig. 7(a). The distorted constellation with a measured EVM of $13 \%$, which is above the FEC limit of $8 \%$, indicates the unsuccessful transmission of the signal over this link. This is caused by the RF beat tone linewidth being too large for the subcarrier baud rate and modulation format being employed. As mentioned earlier, the free space feedback to the MLL results in the reduction of the RF beat tone linewidth. The achieved reduction in the mm-wave carrier linewidth from $\sim 10 \mathrm{kHz}$ without feedback to $\sim 2 \mathrm{kHz}$ with feedback, is shown in Fig. 6(b) from the frequency down converted mm-wave carrier spectrum.

Later the presented optical heterodyne system was tested, when the MLL was operated with free space optical feedback, at the marked operating frequencies from the MLL OFC source. The received signals constellation, shown in Figs. 7(b), results in a measured EVM of $\sim 6 \%$. This shows the importance of generating a mm-wave carrier with low RF linewidth or phase noise. Also, these results indicate the presented systems ability to compensate the PN in the system; while FO is eliminated by use of the OFC. A measured BER of $\sim 1.82 \times 10^{-4}$ was obtained with no penalty due to fiber transmission at a received optical power of $0 \mathrm{dBm}$. In the recent work [24], we have shown the successful transmission of the OFDM signals with subcarrier baud rates of $250 \mathrm{kbaud}$ and higher; indicating the systems capabilities for transmitting the low baud rate/ subcarrier spaced signals required by $5 \mathrm{G}$ standards.

Using the QD-MLL, with feedback, in the mm-wave A-RoF system, we achieved a similar performance to that of our previous demonstrations using a gain switched OFC [18], while eliminating the requirement for an RF synthesizer at the transmitter. Also, any mm-wave or $\mathrm{THz}$ carriers, within the $\mathrm{BW}$ of QD-MLL OFC, can be generated by this process by selecting two lines with the desired frequency spacing for optical heterodyning. This highlights the potential for the deployment of the passively mode-locked lasers mm-wave systems required for provision of multi-carrier A-RoF signals with baud rates compatible with future mobile services. These advantages come at the cost of increased system complexity with the requirement of the free space feedback, WSS, optical amplifier and demultiplexer at the transmitter. However, optical integration could be employed to implement the filtering, amplification, demultiplexing and modulation, thus creating a photonic integrated mm-wave transmitter.

\section{COMPARATIVE DISCUSSION}

Spectrally efficient and cost effective front/back-haul networks, with power efficient and simplistic mm-wave generation, are two key elements to the development of future high speed mobile communication systems. The fronthaul network should facilitate the centralization of the resources along with simplifying the RRH antenna site architecture. In the currently employed CPRI or e-CPRI fronthaul links, digitization of the baseband signal increases the transmission rate over the link, while moving some of the functionalities to the RRH antenna site which requires high speed DAC/ADC. The digitized baseband implementation of current fronthaul transmission links requires remote frequency up-conversion, at the RRH site, for mm-wave operation - limiting the scope for cost saving through hardware centralization.

Meanwhile with the optical heterodyne A-RoF technique, the $\mathrm{RF}$ data signal is directly generated in the mm-wave band as a result of the photo detection process. This simplifies the RRH site as it requires only one high speed photodetector, for $\mathrm{mm}$ wave signal generation, while placing most of the processing components at the C-BBU, as shown in the architecture of Fig. 1. The optical heterodyne A-RoF link aids the centralization

Table. I. Major components of three different FO and PN compensation techniques in an Optical heterodyne A-RoF link for mm-wave signal generation and transmission.

\begin{tabular}{|c|c|c|c|}
\hline Technique & $\begin{array}{c}\text { Mm-wave Analog } \\
\text { Receiver }\end{array}$ & DSP Receiver & $\begin{array}{c}\text { OFC \& Reduced DSP } \\
\text { Receiver }\end{array}$ \\
\hline Hardware at C-BBU & $\begin{array}{c}\text { 2 TLs, IQ MZM, 2 OBPF, } \\
\text { EDFA }\end{array}$ & $\begin{array}{c}\text { 2 TLs, IQ MZM, 2 OBPF, } \\
\text { EDFA }\end{array}$ & $\begin{array}{c}\text { OFC, WSS, 2 EDFA, } \\
\text { DMUX, IQ MZM, 2 OBPF }\end{array}$ \\
\hline Hardware at RRH Site & $70 \mathrm{GHz}$ PIN, PA, Antenna & $70 \mathrm{GHz}$ PIN, PA, Antenna & $70 \mathrm{GHz}$ PIN, PA, Antenna \\
\hline Hardware at Receiver & $\begin{array}{c}\text { 1 Splitter, 2 mm-wave } \\
\text { EBPF, 2 PA, 1 Mixer, } \\
\text { EBPF }\end{array}$ & $\begin{array}{c}\text { PA, mm-wave LO, Mixer, } \\
\text { EBPF }\end{array}$ & $\begin{array}{c}\text { PA, mm-wave LO, Mixer, } \\
\text { EBPF }\end{array}$ \\
\hline $\begin{array}{c}\text { Air Interface Transmission } \\
\text { Requirement }\end{array}$ & $\begin{array}{c}\text { Frequency shifted mm- } \\
\text { wave carrier and data } \\
\text { signal }\end{array}$ & $\begin{array}{c}\text { Frequency shifted } \\
\text { mm-wave signal only }\end{array}$ & Mm-wave signal \\
\hline Software Requirement & None & $\begin{array}{c}\text { S\&C, DD-LMS algorithms } \\
\text { and pilot compensation }\end{array}$ & $\begin{array}{c}\text { DD-LMS algorithm and } \\
\text { pilot compensation }\end{array}$ \\
\hline FO Compensation Limit & $500 \mathrm{MHz}$ (Filter BW limit) & $\sim 140 \mathrm{MHz}$ & No Frequency Offset \\
\hline
\end{tabular}


goal of the fronthaul networks, while retaining the spectral efficiency of the wireless signal over the optical fronthaul link. Also, a laser-free RRH site can be implemented by tapping and reusing the unmodulated carrier, in the optical heterodyne $\mathrm{mm}$ wave A-RoF link, for carrying the uplink data to C-BBU site as demonstrated in our previous work in [39].

In spite of these advantages, the higher phase noise of the photo-generated mm-wave carriers remains one of the hinderance to the deployment of optical heterodyne A-RoF links in the current wireless systems for carrying the small baud rate/subcarrier spacing multicarrier signals that have been standardized for $5 \mathrm{G}$ networks. The three schemes presented in section III, each demonstrate their capabilities to compensate the performance limiting factors in an optical heterodyne $/ \mathrm{mm}$ wave system, i.e. laser frequency offset and phase noise. The complexity of each scheme, in terms of hardware and software requirements, air transmission requirements and FO compensation limitations are outlined in Table I. As mentioned in section III, the first two techniques (i.e. $\mathrm{mm}$-wave analog receiver and DSP receiver) compensate the $\mathrm{FO}$ and $\mathrm{PN}$ at the user side receiver unit, hence most of the additional hardware/software complexity is shifted there. However, in the third technique FO is eliminated at the C-BBU site and PN is compensated at the receiver side, hence its complexity is distributed between C-BBU and receiver.

The analog mm-wave receiver compensates the FO and PN in the same operation using mm-wave cancellation architecture but increases the hardware complexity of the user side receiver unit. However, the "LO-free" frequency down-conversion of the mm-wave data signal reduces the cost of the receiver, while providing a centralized control over most of receiver operations. As the frequency shift in both the photo-mixing generated components ( $\mathrm{Fc}$ and $\mathrm{Fs}$ ) remains the same, irrespective of the laser's relative fluctuations, the level of frequency offset compensated depends on the bandwidths of the EBPF used in the analog mm-wave receiver architecture. Hence, for the presented system a frequency offset up to 500 $\mathrm{MHz}$ (BW of EBPF in LO arm) can be compensated. Our latest work [40] on the analog mm-wave receiver has shown that the $\mathrm{PN}$ capabilities of the receiver is greatly impacted by the chosen $\mathrm{mm}$-wave (carrier and signal) filter design. For the $500 \mathrm{MHz}$ carrier filter bandwidth discussed in this work, [40] indicates that phase noise arising from the interaction of two independent lasers, each exhibiting linewidths in the $\mathrm{MHz}$ range, can be compensated.

Currently, the analog mm-wave receiver can be used in the systems with relaxed spectrum constraints and provision for transmission of mm-wave carrier. It can be used in the systems providing wireless back/front-haul to the RRH antenna sites or fixed user wireless systems, using mm-wave signals which are generated using optical heterodyning of two free running lasers.

The digital compensation of the FO \& PN at the receiver side user unit, transfers the hardware complexity of the analog $\mathrm{mm}$ wave receiver to the digital domain, making the DSP receiver scheme well suited for deployment in the wireless mm-wave systems. External LO induced frequency offset of up to 140 $\mathrm{MHz}$ can be compensated digitally [23] with this technique, showing its capabilities to compensate the FO induced by most of the commercial lasers. Our previous simulation [18] study has concluded that, for an optical heterodyne A-RoF link with independent tunable laser sources (that are frequency locked, but un-correlated in phase), the linewidths of the beating carriers should be less than $\sim 1.5 \%$ of the subcarrier baud rate of the OFDM signal for 64-QAM modulated data. The S\&C algorithm, used in the presented DSP receiver, compensates the FO which is larger than the sub-carrier spacing while the residual FO is manifested as phase rotation in the constellation. This phase rotation is compensated using the DD-LMS algorithm. When both of these processing steps are applied to the received signal, the results, as presented in our previous work [23], follow these above mentioned trends. The DSP approach effectively compensates phase rotations which are inherent to OFDM transmission in the presence of FO. But the extent to which PN specifically attributed to intrinsic laser linewidth, can be overcome, warrants further experimental investigation - including the use of optical sources exhibiting higher linewidth values.

The successful transmission of the mm-wave OFDM signals, with subcarrier spacing as low as $125 \mathrm{kHz}$ [23], over the presented link shows its capabilities to be used in the 5G and beyond $5 \mathrm{G}$ wireless systems. The reduced hardware complexity of the DSP receiver scheme makes it simple and most costefficient for the deployment in most of the mm-wave wireless application.

In order to avoid the transmission of the frequency shifted $\mathrm{mm}$-wave signal through the wireless channel, FO compensation must be done at the C-BBU or RRH site. The use of photonic integrated OFC (based on a QD MLL) at C-BBU and reduced DSP compensation at receiver side, in our third scheme, eliminates the FO while partially shifting the system complexity from user unit to the C-BBU site. The increased complexity, of the C-BBU, due to the requirement of optical filtering, amplification and multiplexing can be reduced by using photonic integration. Further reduction in the complexity can be achieved with recent advances in QD MLL technology [41], which achieve lower linewidth RF beat tones without requirement of free space feedback. The reduced software complexity, with this scheme, for the DSP compensation makes the receiver unit simple and cost efficient. With ideal path matching and high phase correlation between comb lines, OFC based system can successfully transmit very large linewidths. Our previous work [18] has shown that OFC linewidths in the several 10's of MHz can be successfully deployed for optical heterodyne operation with minimal impact of phase noise.

The reduced complexity and ability to generate an exact frequency mm-wave signal along with successful transmission of the 5G envisioned subcarrier spacing OFDM signals makes the MLL OFC and reduced DSP scheme most suitable for deployment in $5 \mathrm{G}$ mobile communication systems. Higher $3 \mathrm{~dB}$ bandwidth, of OFC, and flexibility in the choice of the frequency set for heterodyning makes this scheme more suitable for use in converged optical access network, where the different set of optical tones from the same MLL source can be used for various applications.

In general, the optical heterodyne A-RoF link with compensation based on MLL OFC and reduced DSP provides more practical solution for $\mathrm{mm}$-wave signals generation and transmission. It aids in centralization of the resources while simplifying the user unit and adhering to the wireless channel transmission standards. 


\section{CONCLUSION}

Optical heterodyning A-RoF links provide an efficient platform for the generation and distribution of mm-wave signals for wireless systems. In order to facilitate wide deployment of such fibre-wireless systems, techniques which ease restrictions on relative frequency drift and phase offset between the optical carriers, with minimal additional complexity, are of paramount importance. The results presented in this paper, demonstrate three specific schemes that compensate the mm-wave signals performance limiting factors, i.e. frequency and phase offset in an optical heterodyne A-RoF system. The variable degree of compensation achieved, by different techniques, is demonstrated by successfully transmitting low subcarrier spacing OFDM signal over the link.

The choice of the compensation technique should be made by considering their limitations and the requirements of the application in which the mm-wave A-RoF system will be employed. In general, the optical heterodyne A-RoF system with MLL OFC has the potential to generate a wide range of frequency stable mm-wave carriers from a single device resulting in a simple receiver architecture with reduced DSP requirements. Scaling up the DSP to induce the PN and FO compensation enables the deployment of mm-wave A-RoF systems which can make use of two independent commercially available lasers.

\section{ACKNOWLEDGMENT}

Authors would like to acknowledge PhD student Lakshmi Narayanan V from IIT Madras, India for his feedback regarding this work.

\section{REFERENCES}

[1] J. G. Andrewa et al., "What will 5G Be?," in IEEE Journal on Selected Areas in Communications, vol. 32, no. 6, pp. 1065-1082, June 2014.

[2] China Mobile Research Institute, "C-RAN: The Road Towards Green RAN," White Paper, ver. 2.3, Sep. 2013.

[3] D. Choudhury, "5G wireless and millimeter wave technology evolution: An overview," 2015 IEEE MTT-S International Microwave Symposium, Phoenix, AZ, 2015, pp. 1-4.

[4] CPRI, "Common Public Radio Interface (CPRI); Interface Specification (V7.0)," Tech. Rep., Oct. 2015.

[5] eCPRI, "Common Public Radio Interface (CPRI); eCPRI Interface Specification (V1.0)," Tech. Rep., Aug. 2017.

[6] N. J. Gomes and P. Assimakopoulos, "Optical Fronthaul Options for Meeting 5G Requirements," 20th International Conference on Transparent Optical Networks (ICTON), Bucharest, pp. 1-4, 2018.

[7] S. Cho et al., "Experimental demonstrations of next generation costeffective mobile fronthaul with IFoF technique," Optical Fiber Communications Conference and Exhibition (OFC), Los Angeles, CA, pp. 1-3, 2015 .

[8] C. Han et al., "Performance Improvement of Multi-IFoF-Based Mobile Fronthaul Using Dispersion-Induced Distortion Mitigation with IF Optimization," in Journal of Lightwave Technology, vol. 34, no. 20, pp. 47724778, 15 Oct.15, 2016

[9] A. Delmade et al., "Performance Analysis of Analog IF over Fiber Fronthaul Link With 4G and 5G Coexistance," in Journal of Optical Communications and Networking, 10 (3), pp. 174-182, 2018.

[10] H. Xin, H. He, K. Zhang, S. B. Hussain and W. Hu, "Flexible BasebandUnit Aggregation Enabled by Reconfigurable Multi-IF Over WDM Fronthaul," in IEEE Photonics Journal, vol. 10, no. 1, pp. 1-10, Feb. 2018.
[11] S. Noor, P. Assimakopoulos and N. J. Gomes, "A Flexible Subcarrier Multiplexing System with Analog Transport and Digital Processing for 5G (and Beyond) Fronthaul," in Journal of Lightwave Technology, vol. 37, no. 14, pp. 3689-3700, 15 July15, 2019.

[12] A. Stöhr, "Photonic millimeter-wave generation and its applications in high data rate wireless access," IEEE International Topical Meeting on Microwave Photonics, Montreal, QC, pp. 7-10, 2010.

[13] S. Babiel, R. Chuenchom, A. Stöhr, J. E. Mitchell and Y. Leiba, "Coherent radio-over-fiber (CRoF) approach for heterogeneous wireless-optical networks," Microwave Photonics (MWP) and the 2014 9th Asia-Pacific Microwave Photonics Conference (APMP) 2014 International Topical Meeting on, Sendai, 2014, pp. 25-27.

[14] Tsai C. et al., "Long-reach 60-GHz MMWoF link with free-running laser diodes beating," Scientific Reports 8, 13711, 2018.

[15] Hsiang-Yu Chen, Yu-Chieh Chi, and Gong-Ru Lin, "Remote heterodyne millimeter-wave over fiber based OFDM-PON with master-to-slave injected dual-mode colorless FPLD pair," Opt. Express 23, 22691-22705, 2015.

[16] J. Beas, G. Castanon, I. Aldaya, A. Aragon-Zavala and G. Campuzano, "Millimeter-Wave Frequency Radio over Fiber Systems: A Survey," in IEEE Communications Surveys \& Tutorials, vol. 15, no. 4, pp. 1593-1619, 2013.

[17] Y. Tian, K. Lee, C. Lim and A. Nirmalathas, "Experimental comparison of DSB-SC \& OSSB based $60 \mathrm{GHz}$ radio-over-fiber fronthaul links," 2016 IEEE International Topical Meeting on Microwave Photonics (MWP), Long Beach, CA, pp. 141-144, 2016.

[18] C. Browning et al., "Gain-Switched Optical Frequency Combs for Future Mobile Radio-Over-Fiber Millimeter-Wave Systems," in Journal of Lightwave Technology, vol. 36, no. 19, pp. 4602-4610, Oct.1, 2018.

[19] P. T. Dat et al., "Seamless Convergence of Fiber and Wireless Systems for 5G and Beyond Networks," in Journal of Lightwave Technology, vol. 37, no. 2, pp. 592-605, 15 Jan.15, 2019.

[20] Li Fan et al., "High-purity 60GHz band millimeter-wave generation based on optically injected semiconductor laser under subharmonic microwave modulation," Opt. Express 24, 18252-18265, 2016.

[21] A. Rolland et al., "Non-linear optoelectronic phase-locked loop for stabilization of opto-millimeter waves: towards a narrow linewidth tunable $\mathrm{THz}$ source," Opt. Express 19, 17944-17950, 2011.

[22] Colm Browning et al., "Phase Noise Robust Optical Heterodyne System for Reduced Complexity Millimeter-Wave Analog Radio-over-Fibre,” 2019 European Conference on Optical Communications (ECOC), Dublin, Ireland, M.2.C.4., pp 1-4, 2019.

[23] A. Delmade et al., "OFDM Baud Rate Limitations in an Optical Heterodyne Analog Fronthaul Link using Unlocked Fibre Lasers," 2019 International Topical Meeting on Microwave Photonics (MWP), Ottawa, ON, Canada, pp. 1-4, 2019.

[24] A. Delmade et al., "Quantum Dash Passively Mode Locked Laser for Optical Heterodyne Millimeter-Wave Analog Radio-over-Fiber Fronthaul Systems," in Optical Fiber Communication Conference (OFC) 2020, OSA Technical Digest (Optical Society of America, 2020), paper W2A.41.

[25] T. Kuri and K. Kitayama, "Optical heterodyne detection technique for densely multiplexed millimeter-wave-band radio-on-fiber systems," in Journal of Lightwave Technology, vol. 21, no. 12, pp. 3167-3179, Dec. 2003.

[26] T. Kuri, T. Sakamoto and T. Kawanishi, "Laser-Phase-FluctuationInsensitive Optical Coherent Transmission of 16-Quadrature-AmplitudeModulation Radio-Over-Fiber Signal," in Journal of Lightwave Technology, vol. 34, no. 2, pp. 683-690, 15 Jan.15, 2016.

[27] Y. Shoji, Y. Hashimoto, H. Ogawa, "Fiber-Optic Broadband Signal Distribution Link Based on a Millimeter-Wave Self-Heterodyne Transmission/Optical Remote Heterodyne Detection Technique." in IEICE Trans. Electron., vol. E88-C, no.7, pp.1465-1474, July 2005.

[28] Y. Lin et al., "Characterization of Hybrid InP-TriPleX Photonic Integrated Tunable Lasers Based on Silicon Nitride ( $\mathrm{Si}$ 3N4/SiO2) Microring Resonators for Optical Coherent System," in IEEE Photonics Journal, vol. 10, no. 3, pp. 18, June 2018 
[29] T.M. Schmidl and C. Cox, "Robust Frequency and Timing Synchronization for OFDM," IEEE Trans. On Communications, 45 (12), pp. 1613-1621, 1997.

[30] Jiadi Wuet al., "A robust and efficient frequency offset correction algorithm with experimental verification for coherent optical OFDM system," Journal of Lightwave Technology,33, pp. 3801-3807, 2015.

[31] H. Y. Rha, C. J. Youn, Y. Kwon and H. Choi, "Real-Time Digital Signal Processing for High-Speed Coherent Optical OFDM Synchronization," in Journal of Lightwave Technology, vol. 33, no. 11, pp. 2294-2302, 1 June, 2015.

[32] A. Delmade et al., "Digital Frequency/Phase Offset Correction for $60 \mathrm{GHz}$ OFDM Radio-over-Fibre with Unlocked Fibre Lasers", in ECOC, paper P74, September 2019.

[33] H. Shams et al., "100 Gb/s Multicarrier THz Wireless Transmission System with High Frequency Stability Based on A Gain-Switched Laser Comb Source," in IEEE Photonics Journal, vol. 7, no. 3, pp. 1-11, Art no. 7902011, June 2015.

[34] H. H. Elwan et al., "Impact of Laser Mode Partition Noise on Optical Heterodyning at Millimeter-Wave Frequencies," in Journal of Lightwave Technology, vol. 34, no. 18, pp. 4278-4284, 15 Sept.15, 2016.

[35] G. Duan et al., "High performance InP-based quantum dash semiconductor mode-locked lasers for optical communications," in Bell Labs Technical Journal, vol. 14, no. 3, pp. 63-84, Fall 2009.

[36] Ricardo Rosales et al., "High performance mode locking characteristics of single section quantum dash lasers," Opt. Express 20, 8649-8657, 2012.

[37] T. Verolet et al., "Mode Locked Laser Phase Noise Reduction under Optical Feedback for Coherent DWDM Communication," in Journal of Lightwave Technology.

[38] K. Merghem et al., "Narrow linewidth frequency comb source based on self-injected quantum-dash passively mode-locked laser," Conference on Lasers and Electro-Optics (CLEO), San Jose, CA, paper SW1C.5, 2017.

[39] A. Kaszubowska-Anandarajah et al., "Bidirectional fiber transmission of $\mathrm{mmW}$ signals using remote downconversion and wavelength reuse," in Conference on Lasers and Electro-Optics, OSA Technical Digest (Optical Society of America, 2019), paper SM4G.2.

[40] D. Dass et al., "Analysis of Phase Noise in a Hybrid Photonic/MillimetreWave System for Single and Multi-Carrier Radio Applications," Appl. Sci., 10, $5800,2020$.

[41] Guocheng Liu et al., "Passively mode-locked quantum dash laser with an aggregate 5.376 Tbit/s PAM-4 transmission capacity," Opt. Express 28, 45874593, 2020. 